\title{
Exogenous Endophthalmitis in Diabetic Patients: A Systemic Review
}

\author{
Georges M. El-Mollayess, Joanna S. Saadeh, and Haytham I. Salti \\ The Department of Ophthalmology, American University of Beirut Medical Center, P.O. Box 11-0236, Beirut 1107 2020, Lebanon
}

Correspondence should be addressed to Haytham I. Salti, hs06@aub.edu.lb

Received 2 August 2012; Accepted 26 August 2012

Academic Editors: N. Ardjomand and I. J. Wang

Copyright ( $) 2012$ Georges M. El-Mollayess et al. This is an open access article distributed under the Creative Commons Attribution License, which permits unrestricted use, distribution, and reproduction in any medium, provided the original work is properly cited.

\begin{abstract}
Diabetes mellitus is a systemic disease that increases the risk of infections. Exogenous endophthalmitis is an inflammatory disease to which diabetic patients are more predisposed to than nondiabetic patients undergoing any intraocular intervention. This might be because of the change in the immune and inflammatory factors that intervene in wound healing and in the bacterial flora of the ocular adnexa. We conducted a literature review to assess the risk of exogenous endophthalmitis in diabetic patients undergoing cataract extraction, pars plana vitrectomy, and intravitreal injections and to check whether its treatment differ from in nondiabetics. We found that diabetic patients are more predisposed to virulent organisms and that the incidence of ophthalmic symptoms was not substantially different in diabetic versus nondiabetic patients. Regarding treatment, all patients with light perception should receive pars plana vitrectomy, while those with hand motion and better vision should be given an intravitreal antibiotics injection. Some authors recommend vitrectomy to diabetic patients with even counting figure vision.
\end{abstract}

\section{Introduction}

Infectious endophthalmitis is defined as the infestation of the intraocular compartment by microorganisms. It represents one of the most severe and potentially devastating inflammatory reactions seen in the eye and it often results in irreversible visual loss $[1,2]$. Based on the site of entry of this agent, endophthalmitis can be divided into an infection of either exogenous or endogenous origin. While the former condition most commonly occurs after intraocular surgery or trauma, the latter form is believed to be linked to septicemia. Postoperative bacterial endophthalmitis is a rare, but severe complication of intraocular surgery. The infecting bacteria by replication and release of toxins can damage the intraocular structures, and the inflammatory reaction of the host seems to contribute even further to the damage [3].

Patients with diabetes mellitus (DM) known to have an impaired immune response may be at a higher risk for developing postoperative infections. Effectively at the cellular and humoral levels, there is suboptimal response to different antigens in addition to altered phagocytic capabilities [47]. Moreover, it is established that impaired neutrophil bactericidal function is strongly associated with poor glycemic control $[8,9]$. Furthermore, the tear film, the first immunological barrier for the ocular system, is altered in patients with DM. There is documented decrease in the breakup time and an established decrease in Schirmer's test [10]. Finally, it has been demonstrated that diabetes mellitus alters the corneal epithelial basement membrane resulting in basal cell degeneration manifested clinically as a superficial punctate keratitis and breakdown of the barrier function of this front line epithelium resulting in greater fragility of the eyeball [11-17].

These deficiencies in the protective features inherent to the eye seem to all associate with the duration of the disease and the serum level of glycosylated hemoglobin HbAlc [18, 19]. Elevated levels of of glucose seem to impair epidermal growth factor receptor (EGFR) signaling and suppress basal cell multiplication and wound-induced AKT phosphorylation [20-22]. This anomaly results in delayed healing of the epithelial defect, which may be associated with sightthreatening complications, such as stromal opacities, surface irregularities, microbial keratitis, and increased risk of acute postoperative endophthalmitis due to delayed and improper wound closure [22]. Independent of all the above and with 
TABLE 1: Visual outcome and symptoms in diabetics versus nondiabetics.

\begin{tabular}{lcc}
\hline & Diabetics & Nondiabetics \\
\hline Posttreatment visual outcome & $(\%)$ & $(\%)$ \\
$\geq 5 / 200$ & 79.6 & 90 \\
$\geq 20 / 100$ & 55.6 & 77.4 \\
$\geq 20 / 40$ & 38.9 & 55.3 \\
Anterior segment characteristics & $(\%)$ & $(\%)$ \\
Cataract wound normal & 82.8 & 82.0 \\
Hypopyon & 89.7 & 85.1 \\
Rubeosis & 8.6 & 1.9 \\
Bacterial growth (risk) & & - \\
Gram+ & Increased & \\
Gram- & Same & $22 \%$ \\
Virulent organisms & $26 \%$ & $8.6 \%$ \\
Incidence of retinal detachment (Posttreatment) & $6.9 \%$ & $27.8 \%$ \\
Early procedure performed & $20.7 \%$ & $31 \%$ \\
Late procedure performed & & \\
\hline
\end{tabular}

the raised levels of glucose in the skin, mucous membranes and the tear film of patients with DM, microorganisms growth seems definitely promoted.

\section{Epidemiology}

The reported incidence of postoperative endophthalmitis varies by the specific surgical procedure, but overall the occurrence has declined substantially in the past century. The incidence of endophthalmitis after cataract surgery has decreased from approximately $10 \%$ in the late 1800 s to $0.58 \%$ in the mid-1900s to $0.09 \%$ in the early 1990 s to $0.04 \%$ during the period 1995 to 2001 [1, 23-26]. Rates of endophthalmitis after conventional pars plana vitrectomy (PPV) have further decreased over the past 25 years. Since Ho and Tolentino reported an endophthalmitis rate as high as $0.15 \%$ after PPV in 1984, in subsequent years, the incidence has decreased to a range from $0.018 \%$ to $0.07 \%$ [25-32]. The question that whether sutureless vitrectomy increases the risk of endophthalmitis is still controversial [31, 33, 34]. Past literature suggests that subjects with diabetes have an increased tendency, after cataract surgery, to develop endophthalmitis $[1,32,35,36]$. A plethora of reports show that approximately 14 to $21 \%$ of postoperative patients who develop endophthalmitis are diabetic [37-40].

\section{Symptoms and Presentation}

The incidence of ophthalmic symptoms was not substantially different in diabetic versus nondiabetic subjects, especially that patients presenting with ocular pain are nearly as frequent in both types of subjects: the diabetic and the nondiabetic. The median number of days from onset of symptoms to presentation also did not differ in both types of patients (on the average 4 days) [38]. The differences were essentially a trend towards a more opaque media in diabetic subjects upon examination than in the nondiabetics. A retinal vessel could not be seen with indirect ophthalmoscopy at presentation in $90 \%$ of diabetics, versus $77 \%$ in nondiabetic patients. Also, the incidence of rubeosis at presentation was $8.6 \%$ in diabetics versus $1.9 \%$ in the nondiabetics [38]. Table 1 summarizes the frequency of symptoms in diabetic patients and in nondiabetic patients.

\section{Types of Isolated Microorganisms and Antibiotics Sensitivity}

Postoperative endophthalmitis is most often associated with gram-positive organisms $(75 \%-80 \%)$, next in frequency are gram-negative organisms $(15 \%-29 \%)$, and least often fungi $(3 \%-13 \%)$. When cultures were obtained from nondiabetic patients they were likely to show no growth in as many as twice the number noted in cultures from diabetic subjects that is at $33 \%$ versus $15 \%$, respectively. In addition, the microorganisms that grew from eyes of diabetic subjects had a preponderance of more virulent organisms at $26 \%$ versus $22 \%$ isolated from eyes in nondiabetic patients [38]. The most common organism isolated from diabetic patients with acute endophthalmitis is coagulase-negative Staphylococcus [37]. In another study, both type 1 and type 2 diabetic patients grew staphylococcus epidermis and staphylococcus aureus at $11.79 \%$ and $11.7 \%$, respectively for type 1 , and $24.2 \%$ and $21.2 \%$ for type 2 [10]. There was a trend for a higher preponderance of coagulase-negative staphylococcal isolates in patients who had preexisting diabetic retinopathy [10]. Phillips and Tasman found a higher prevalence of gram-negative isolates of Staph organisms among their patients. Remarkably none of their patients had gramnegative endophthalmitis [41]. Of note, is the conjunctival flora of diabetic subjects differed from that isolated from nondiabetic subjects [10].

As for resistance to antibiotics, very few reports exist in the literature comparing resistance to antibiotics among different microorganisms isolated from patients with diabetes as opposed to those without. On the one hand, resistance 


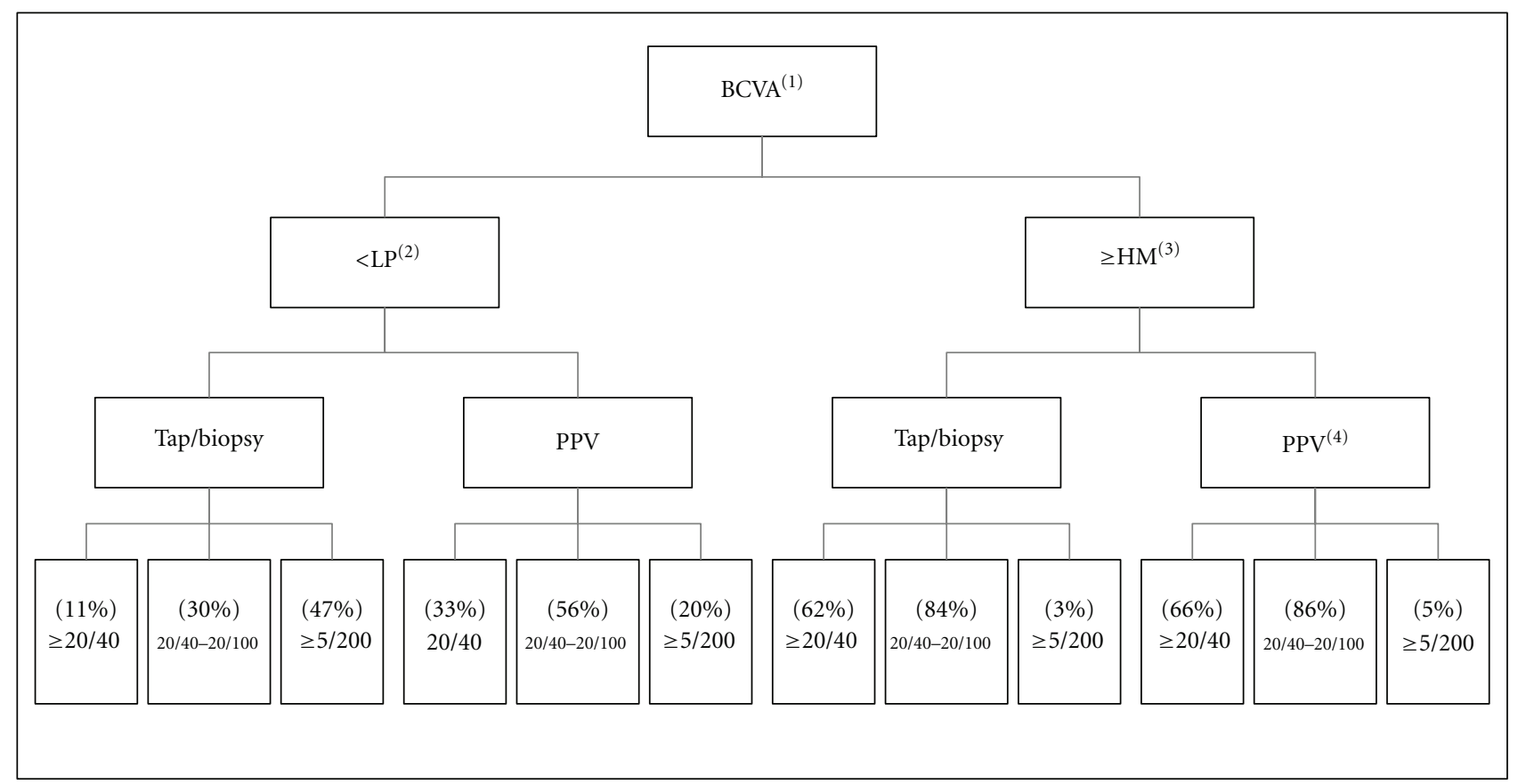

Figure 1: Visual outcome of endophthalmitis vitrectomy study. (1) Best corrected visual acuity, (2) light perception, (3) hand motion, (4) pars plana vitrectomy.

was observed and reported to penicillin, ampicillin, and tetracycline in S. aureus isolates, on the other hand resistance to vancomycin was absent, thus singling out this antibiotic as the most effective therapeutic option [10].

\section{Treatment}

Endophthalmitis still poses a challenge to both the timing and the modality of treatment. To date, the largest series that has directed the standard of care remains to be the EVS, which in summary recommends the following. In eyes with LP vision or better, tap and injection were as favored as the use of pars plana vitrectomy plus tap. While in eyes with LP vision or worse the group that underwent the PPV fared better than patients who received tap with intravitreal antibiotics only. Subanalysis of the patients with diabetes in the EVS cohort was inconclusive although it suggested a more favorable outcome for patients who underwent a vitrectomy regardless of their visual outcome [42] (Figure 1: summary of the study is included in the flowchart).

The use of intravitreal dexamethasone along with the antibiotics injection is still controversial. On the one hand, if intravitreal steroids are given early enough in the course of endophthalmitis, there might be some benefit for better outcome in endophthalmitis through a reduction in the inflammatory response to the infectious organism [43-46]. In one study done on nondiabetic patients who developed endophthalmitis, there was a trend towards a better visual outcome in patients with suspected bacterial endophthalmitis when treated with a combination of intravitreal antibiotics and dexamethasone [47]. On the other hand, another report demonstrated that intravitreal steroids may not be effective for acute endophthalmitis besides and it is important to be cautious using it, keeping in mind its potential toxicity and blunting of the immune response that is necessary to combat infections [48]. Jett et al. noted that combining intravitreal steroids with antibiotics may have beneficial effects in eyes infected with only bacteria that are nontoxin-producing and are less virulent; they reported that manipulation of the timing of dexamethasone administration did not have any significant effect on the treatment outcome [49]. It is obvious that studies on the use and timing of intravitreal steroids in diabetic patients with endophthalmitis are badly needed to establish a solid basis for treatment that would constitute standardized care.

\section{Systemic Antibiotics}

Prior results from EVS demonstrated that systemic antibiotics were not effective in the management of acute postcataract extraction endophthalmitis [40].

\section{Visual Outcome after Treatment}

The outcome of vision in patients with diabetes treated for endophthalmitis is usually less successful than in patients with no diabetes. Phillips and Tasman found that $26 \%$ of patients with diabetes achieved a final postoperative visual acuity equal or slightly more than $20 / 200$ in contrast to $59 \%$ in patients without diabetes. Additionally, $30 \%$ of these diabetic patients failed to have not even light perception vision [41]. In an exploratory analysis among patients in the Endophthalmitis Vitrectomy Study (EVS), 58 out of 420 
(13.8\%) eyes of diabetic patients were analysed over a 9month period, $80 \%$ achieved visual acuity of $5 / 200$ or greater in contrast to $90 \%$ in patients who had no diabetes. Along this trend, $55 \%$ and $39 \%$ of subjects with diabetes achieved a final visual acuity equal or slightly greater than, respectively, $20 / 100$ or greater and $20 / 40$ or greater, compared to $77 \%$ and $55 \%$ in nondiabetic patients, respectively. As to patients who presented with better than LP vision, diabetic subjects achieved 20/40 more often when vitrectomy was applied (57\%) than with tap/biopsy (40\%) [38, 40]. No operative complications were observed in either group although early postoperative interventions were significantly higher in the group of diabetic patients $(20.7 \%)$. However, the statistical power is very low because of the small number of subjects with diabetes [38]. Thus, until a large clinical trial studying treatment outcome in diabetic patients with better than LP vision is done, it is recommended that initial vitrectomy or tap/biopsy are reasonable approaches for diabetic patients with better than LP vision [50].

\section{Endophthalmitis and Intravitreal Injection}

Endophthalmitis, although a rare complication of intravitreal injection, is a serious and clinically relevant concern. This is because there are situations in which the frequency and number of injections are obligatory to avoid potential loss of vision, which otherwise may be permanent in spite of prompt and appropriate management [51]. Its incidence per injection of either bevacizumab, ranibizumab, pegaptanib, or triamcinolone ranges from $0.009 \%$ to $1.9 \%$ [51-56]. The pathogens most commonly isolated from intravitreal cultures are Staphylococcus epidermidis and staphylococci coagulase-negative [57-59]. Optimum management of the ocular surface before, during, and after intravitreal injections remains controversial. A topical combination of povidoneiodine is the only preoperative substance proven in a randomized clinical trial to reduce the risk of endophthalmitis after intraocular surgery $[58,60]$. In the 2008 ASRS meeting, it has been suggested that $40 \%$ of retina specialists use topical antibiotics before anti-vascular endothelial growth factor intravitreal injections, and $86 \%$ use topical antibiotics after anti vascular endothelial growth factor intravitreal injections. In a report from the DRCR.NET, a low rate of endophthalmitis can be achieved by means of a protocol that includes use of topical povidone-iodine, a sterile lid speculum, and topical anesthetic, but does not require topical antibiotics, sterile gloves, or a sterile drape [58]. Achievement of a low rate of endophthalmitis postintravitreal injections does not require topical antibiotic prophylaxis a day before or after the injection [58].

\section{Endophthalmitis after PPV}

In a 20-year retrospective review of patients who developed acute onset endophthalmitis after pars plana vitrectomy at Bascom Palmer Eye Institute, 5 out of 6 patients had diabetes. Patients presented with an initial visual acuity ranging from $20 / 300$ to light perception. Visual outcome postsurgery was poor with 4 out of 6 patients having a final vision of light perception or worse. Initial advanced retinopathy stages that these patients had might have hampered any improvement in vision. This is further supported by the Post-Vitrectomy Endophthalmitis Study, where $61 \%$ of the patients $(11 / 18)$ had diabetes mellitus [32].

The visual outcome of postvitrectomy endophthalmitis is usually poor [28, 32]. Eyes undergoing sutureless vitrectomy may have an increased risk of infectious endophthalmitis compared to 20-gauge vitrectomy. Series reported earlier suggest that there was an increased risk of endophthalmitis, in contrast to more recent series, which so far report mixed results [31, 34, 61-69]. Meanwhile, variable hypotheses have been advanced to explain why sutureless transconjunctival PPV may lead to a higher rate of postoperative endophthalmitis. Some theories relate it to a lack of complete wound closure. Ultrasound biomicroscopy demonstrates that 25gauge wounds reappose within 2 weeks $[70,71]$. We do not know whether the level of ischemia caused by diabetes interferes in the proper wound healing of the sclera and the development of fibrovascular ingrowth into the vitreous base. Some suggests leaving air- or gas-filled vitreous cavity would allow sclera wound apposition secondary to the surface tension that develops at the wound interface $[34,51$, 72]. Others propose that lower infusion rates are a feature of sutureless vitrectomy, and the reduced influx and efflux of fluid may allow a greater bacterial inoculum to remain in the eye [73]. In addition, less vitreous gel is removed during sutureless PPV versus 20-gauge PPV and the residual vitreous skirt may facilitate bacterial adherence and sequester bacteria $[74,75]$. Future retrospective or prospective trials need to take into consideration certainly several factors.

Treatment of postvitrectomy endophthalmitis is analogous to treatment of other types of postoperative endophthalmitis. The best treatment option for gas-filled eyes with postvitrectomy endophthalmitis is not yet established. Intravitreal antibiotics are generally recommended in such eyes, but the dosage and specific drug may vary. The recommended standard dose age of intravitreal antibiotics could be beneficial in even up to $50 \%$ of gas-filled eyes [28].

\section{Diabetic Retinopathy and Endophthalmitis}

Diabetic retinopathy may become worse with any inflammatory process because eyes in diabetic subjects are susceptible to usually upsurging in some inflammatory factors. Huamonte and associates reported two cases of progression of diabetic retinopathy associated with the inflammatory processes typically associated with sarcoidosis [76]. This concept is further supported by the progression of diabetic retinopathy after cataract extraction. Several authors have shown that retinopathy progression after cataract surgery strongly correlates with the level of preoperative retinopathy [77-82]. Patients with preexisting diabetic retinopathy may be at increased risk for rapid retinopathy progression and a poorer visual outcome after endophthalmitis [37, 83]. So, the visual outcome after endophthalmitis treatment is highly dependent on the level of the damage caused by diabetic retinopathy before the development of endophthalmitis; this prompted the specialists in the field to recommend 
close monitoring of diabetic patients after endophthalmitis, whether or not they have preexisting DR.

In conclusion, although no large-scale study has looked exclusively at subjects with diabetes and endophthalmitis, analysis of the subgroup of patients with diabetes in studies comprising both (patients with diabetes and patients without) suggest that virulence is worse in the former group, growth of organisms is faster and a more aggressive treatment bares a better result. Even though no study has addressed the sutureless trend and the higher risk of endophthalmitis in patients with diabetes, it is wise to consider more conventional wound closures. Special care and more forceful management are warranted in every step. Finally, diabetic retinopathy, if preexisting, progresses to worse, therefore, more frequent retina examinations are advised in this particular subgroup.

\section{Conflict of Interests}

The authors have no financial interest in any product mentioned in the study.

\section{References}

[1] H. M. Kattan, H. W. Flynn, S. C. Pflugfelder, C. Robertson, and R. K. Forster, "Nosocomial endophthalmitis survey: current incidence of infection after intraocular surgery," Ophthalmology, vol. 98, no. 2, pp. 227-238, 1991.

[2] D. R. May and G. A. Peyman, "Endophthalmitis after vitrectomy," American Journal of Ophthalmology, vol. 81, no. 4, pp. 520-521, 1976.

[3] M. C. Callegan, M. Engelbert, D. W. Parke, B. D. Jett, and M. S. Gilmore, "Bacterial endophthalmitis: epidemiology, therapeutics, and bacterium-host interactions," Clinical Microbiology Reviews, vol. 15, no. 1, pp. 111-124, 2002.

[4] M. P. Moutschen, A. J. Scheen, and P. J. Lefebvre, "Impaired immune responses in diabetes mellitus: analysis of the factors and mechanisms involved. Relevance to the increased susceptibility of diabetic patients to specific infections," Diabete et Metabolisme, vol. 18, no. 3, pp. 187-201, 1992.

[5] A. S. Al-Kassab and S. Raziuddin, "Immune activation and $\mathrm{T}$ cell subset abnormalities in circulation of patients with recently diagnosed type I diabetes mellitus," Clinical and Experimental Immunology, vol. 81, no. 2, pp. 267-271, 1990.

[6] C. K. Abrass, "Fc receptor-mediated phagocytosis: abnormalities associated with diabetes mellitus," Clinical Immunology and Immunopathology, vol. 58, no. 1, pp. 1-17, 1990.

[7] G. Carnazzo, G. Mirone, A. Turturici et al., "Pathophysiology of the immune system in elderly subjects with or without diabetes and variations after recombinant interleukin-2," Archives of Gerontology and Geriatrics, vol. 9, no. 2, pp. 163-180, 1989.

[8] T. C. Alba-Loureiro, C. D. Munhoz, J. O. Martins et al., "Neutrophil function and metabolism in individuals with diabetes mellitus," Brazilian Journal of Medical and Biological Research, vol. 40, no. 8, pp. 1037-1044, 2007.

[9] A. Mowat and J. Baum, "Chemotaxis of polymorphonuclear leukocytes from patients with diabetes mellitus," New England Journal of Medicine, vol. 284, no. 12, pp. 621-627, 1971.

[10] H. Bilen, O. Ates, N. Astam, H. Uslu, G. Akcay, and O. Baykal, "Conjunctival flora in patients with type 1 or type 2 diabetes mellitus," Advances in Therapy, vol. 24, no. 5, pp. 1028-1035, 2007.

[11] J. Friend, Y. Ishii, and R. A. Thoft, "Corneal epithelial changes in diabetic rats," Ophthalmic Research, vol. 14, no. 4, pp. 269278, 1982.

[12] H. R. Taylor and R. A. Kimsey, "Corneal epithelial basement membrane changes in diabetes," Investigative Ophthalmology and Visual Science, vol. 20, no. 4, pp. 548-553, 1981.

[13] D. T. Azar, S. J. Spurr-Michaud, A. S. Tisdale, and I. K. Gipson, "Altered epithelial-basement membrane interactions in diabetic corneas," Archives of Ophthalmology, vol. 110, no. 4, pp. 537-540, 1992.

[14] I. S. Zagon, J. W. Sassani, and P. J. McLaughlin, "Insulin treatment ameliorates impaired corneal reepithelialization in diabetic rats," Diabetes, vol. 55, no. 4, pp. 1141-1147, 2006.

[15] K. Inoue, K. Okugawa, S. Amano et al., "Blinking and superficial punctate keratopathy in patients with diabetes mellitus," Eye, vol. 19, no. 4, pp. 418-421, 2005.

[16] U. Rehany, Y. Ishii, M. Lahav, and S. Rumelt, "Ultrastructural changes in corneas of diabetic patients: an electron-microscopy study," Cornea, vol. 19, no. 4, pp. 534-538, 2000.

[17] J. S. Saini and B. Khandalavla, "Corneal epithelial fragility in diabetes mellitus," Canadian Journal of Ophthalmology, vol. 30, no. 3, pp. 142-146, 1995.

[18] B. D. Manning and L. C. Cantley, "AKT/PKB signaling: navigating downstream," Cell, vol. 129, no. 7, pp. 1261-1274, 2007.

[19] D. J. Panka, M. B. Atkins, and J. W. Mier, "Targeting the mitogen-activated protein kinase pathway in the treatment of malignant melanoma," Clinical Cancer Research, vol. 12, no. 7, pp. 2371s-2375s, 2006.

[20] G. N. Rao, "Dr. P. Siva Reddy Oration. Diabetic keratopathy," Indian Journal of Ophthalmology, vol. 35, no. 5-6, pp. 16-36, 1987.

[21] K. P. Xu, Y. Li, A. V. Ljubimov, and F. S. X. Yu, "High glucose suppresses epidermal growth factor receptor/phosphatidylinositol 3-kinase/akt signaling pathway and attenuates corneal epithelial wound healing," Diabetes, vol. 58, no. 5, pp. 1077-1085, 2009.

[22] K. Xu and F. S. Yu, "Impaired epithelial wound healing and EGFR signaling pathways in the corneas of diabetic rats," Investigative Ophthalmology \& Visual Science, vol. 52, no. 6, pp. 3301-3308, 2011.

[23] J. C. Javitt, S. Vitale, J. K. Canner et al., "National outcomes of cataract extraction: endophthalmitis following inpatient surgery," Archives of Ophthalmology, vol. 109, no. 8, pp. 10851089, 1991.

[24] H. F. Allen and A. B. Mangiaracine, "Bacterial endophthalmitis after cataract extraction: a study of 22 infections in 20,000 operations," Archives of ophthalmology, vol. 72, pp. 454-462, 1964.

[25] T. M. Aaberg, H. W. Flynn, J. Schiffman, and J. Newton, "Nosocomial acute-onset postoperative endophthalmitis survey: a 10-year review of incidence and outcomes," Ophthalmology, vol. 105, no. 6, pp. 1004-1010, 1998.

[26] C. W. G. Eifrig, H. W. Flynn Jr., I. U. Scott, and J. Newton, "Acute-onset postoperative endophthalmitis: review of incidence and visual outcomes (1995-2001)," Ophthalmic Surgery and Lasers, vol. 33, no. 5, pp. 373-378, 2002.

[27] P. C. Ho and F. I. Tolentino, "Bacterial endophthalmitis after closed vitrectomy," Archives of Ophthalmology, vol. 102, no. 2, pp. 207-210, 1984.

[28] C. W. G. Eifrig, I. U. Scott, H. W. Flynn, W. E. Smiddy, and J. Newton, "Endophthalmitis after pars plana vitrectomy: incidence, causative organisms, and visual acuity outcomes," 
American Journal of Ophthalmology, vol. 138, no. 5, pp. 799802, 2004.

[29] T. Sakamoto, H. Enaida, T. Kubota et al., "Incidence of acute endophthalmitis after triamcinolone-assisted pars plana vitrectomy," American Journal of Ophthalmology, vol. 138, no. 1, pp. 137-138, 2004.

[30] S. Zhang, X. Ding, J. Hu, and R. Gao, "Clinical features of endophthalmitis after vitreoretinal surgery," Yan Ke Xue Bao, vol. 19, no. 1, pp. 39-43, 2003.

[31] D. Y. Kunimoto and R. S. Kaiser, "Incidence of Endophthalmitis after 20- and 25-Gauge Vitrectomy," Ophthalmology, vol. 114, no. 12, pp. 2133-2137, 2007.

[32] S. M. Cohen, H. W. Flynn, T. G. Murray et al., "Endophthalmitis after pars plana vitrectomy," Ophthalmology, vol. 102, no. 5, pp. 705-712, 1995.

[33] I. U. Scott, H. W. Flynn, S. Dev et al., "Endophthalmitis after 25-gauge and 20-gauge pars plana vitrectomy: incidence and outcomes," Retina, vol. 28, no. 1, pp. 138-142, 2008.

[34] B. Parolini, F. Romanelli, G. Prigione, and G. Pertile, "Incidence of endophthalmitis in a large series of 23-gauge and 20gauge transconjunctival pars plana vitrectomy," Graefe's Archive for Clinical and Experimental Ophthalmology, vol. 247, no. 7, pp. 895-898, 2009.

[35] P. G. Montan, G. Koranyi, H. E. Setterquist, A. Stridh, B. T. Philipson, and K. Wiklund, "Endophthalmitis after cataract surgery: risk factors relating to technique and events of the operation and patient history: a retrospective case-control study," Ophthalmology, vol. 105, no. 12, pp. 2171-2177, 1998.

[36] I. U. Scott, H. W. Flynn, and W. Feuer, "Endophthalmitis after secondary intraocular lens implantation: a case- control study," Ophthalmology, vol. 102, no. 12, pp. 1925-1931, 1995.

[37] S. Dev, J. S. Pulido, H. H. Tessler et al., "Progression of diabetic retinopathy after endophthalmitis," Ophthalmology, vol. 106, no. 4, pp. 774-781, 1999.

[38] B. H. Doft, S. R. Wisniewski, S. F. Kelsey, and S. GroerFitzgerald, "Diabetes and postcataract extraction endophthalmitis," Current Opinion in Ophthalmology, vol. 13, no. 3, pp. 147-151, 2002.

[39] I. S. Jain, C. L. Luthra, and T. Das, "Diabetic retinopathy and its relation to errors of refraction," Archives of Ophthalmology, vol. 77, no. 1, pp. 59-60, 1967.

[40] B. Doft, T. Nakamura, A. F. Walonker et al., "Results of the endophthalmitis vitrectomy study: a randomized trial of immediate vitrectomy and of intravenous antibiotics for the treatment of postoperative bacterial endophthalmitis," Archives of Ophthalmology, vol. 113, no. 12, pp. 1479-1496, 1995.

[41] W. B. Phillips and W. S. Tasman, "Postoperative endophthalmitis in association with diabetes mellitus," Ophthalmology, vol. 101, no. 3, pp. 508-518, 1994.

[42] B. H. Doft, "Treatment of postcataract extraction endophthalmitis: a summary of the results from the endophthalmitis vitrectomy study," Archives of Ophthalmology, vol. 126, no. 4, pp. 554-556, 2008.

[43] R. O. Graham and G. A. Peyman, "Intravitreal injection of dexamethasone. Treatment of experimentally induced endophthalmitis," Archives of Ophthalmology, vol. 92, no. 2, pp. 149-154, 1974.

[44] S. S. Park, N. Samiy, K. Ruoff, D. J. D’Amico, and A. S. Baker, "Effect of intravitreal dexamethasone in treatment of pneumococcal endophthalmitis in rabbits," Archives of Ophthalmology, vol. 113, no. 10, pp. 1324-1329, 1995.

[45] L. K. Mao, H. W. Flynn, D. Miller, and S. C. Pflugfelder, "Endophthalmitis caused by Staphylococcus aureus," American Journal of Ophthalmology, vol. 116, no. 5, pp. 584-589, 1993.
[46] W. D. Irvine, H. W. Flynn, D. Miller, and S. C. Pflugfelder, "Endophthalmitis caused by gram-negative organisms," Archives of Ophthalmology, vol. 110, no. 10, pp. 1450-1454, 1992.

[47] I. M. Gan, L. C. Ugahary, J. T. van Dissel et al., "Intravitreal dexamethasone as adjuvant in the treatment of postoperative endophthalmitis: a prospective randomized trial," Graefe's Archive for Clinical and Experimental Ophthalmology, vol. 243, no. 12, pp. 1200-1205, 2005.

[48] G. K. Shah, J. D. Stein, S. Sharma et al., "Visual outcomes following the use of intravitreal steroids in the treatment of postoperative endophthalmitis," Ophthalmology, vol. 107, no. 3, pp. 486-489, 2000.

[49] B. D. Jett, H. G. Jensen, R. V. Atkuri, and M. S. Gilmore, "Evaluation of therapeutic measures for treating endophthalmitis caused by isogenic toxin-producing and toxin-nonproducing Enterococcus faecalis strains," Investigative Ophthalmology and Visual Science, vol. 36, no. 1, pp. 9-15, 1995.

[50] B. H. Doft, S. R. Wisniewski, S. F. Kelsey, and S. G. Fitzgerald, "Diabetes and postoperative endophthalmitis in the endophthalmitis vitrectomy study," Archives of Ophthalmology, vol. 119, no. 5, pp. 650-656, 2001.

[51] L. L. Cavalcante, M. L. Cavalcante, T. G. Murray et al., "Intravitreal injection analysis at the Bascom palmer eye institute: evaluation of clinical indications for the treatment and incidence rates of endophthalmitis," Clinical Ophthalmology, vol. 4, no. 1, pp. 519-524, 2010.

[52] D. R. Fintak, G. K. Shah, K. J. Blinder et al., "Incidence of endophthalmitis related to intravitreal injection of bevacizumab and ranibizumab," Retina, vol. 28, no. 10, pp. 1395-1399, 2008.

[53] J. O. Mason, M. F. White, R. M. Feist et al., "Incidence of acute onset endophthalmitis following intravitreal bevacizumab (Avastin) injection," Retina, vol. 28, no. 4, pp. 564-567, 2008.

[54] S. Pilli, A. Kotsolis, R. F. Spaide et al., "Endophthalmitis associated with intravitreal anti-vascular endothelial growth factor therapy injections in an office setting," American Journal of Ophthalmology, vol. 145, no. 5, pp. 879-882, 2008.

[55] I. U. Scott and H. W. Flynn, "Reducing the risk of endophthalmitis following intravitreal injections," Retina, vol. 27, no. 1, pp. 10-12, 2007.

[56] G. M. El-Mollayess, B. N. Noureddine, and Z. F. Bashshur, "Bevacizumab and neovascular age related macular degeneration: pathogenesis and treatment," Seminars in Ophthalmology, vol. 26, no. 3, pp. 69-76, 2011.

[57] K. S. Klein, M. K. Walsh, T. S. Hassan et al., "Endophthalmitis after anti-VEGF injections," Ophthalmology, vol. 116, no. 6, p. 1225, 2009.

[58] A. R. Bhavsar, J. M. Googe, C. R. Stockdale et al., "Risk of endophthalmitis after intravitreal drug injection when topical antibiotics are not required: the diabetic retinopathy clinical research network laser-ranibizumab-triamcinolone clinical trials," Archives of Ophthalmology, vol. 127, no. 12, pp. 15811583, 2009.

[59] T. Diago, C. A. McCannel, S. J. Bakri, J. S. Pulido, A. O. Edwards, and J. M. Pach, "Infectious endophthalmitis after intravitreal injection of antiangiogenic agents," Retina, vol. 29, no. 5, pp. 601-605, 2009.

[60] M. G. Speaker and J. A. Menikoff, "Prophylaxis of endophthalmitis with topical povidone-iodine," Ophthalmology, vol. 98, no. 12, pp. 1769-1775, 1991.

[61] S. R. J. Taylor and G. W. Aylward, "Endophthalmitis following 25-gauge vitrectomy," Eye, vol. 19, no. 11, pp. 1228-1229, 2005. 
[62] M. Taban, R. L. Ufret-Vincenty, and J. E. Sears, "Endophthalmitis after 25-gauge transconjunctival sutureless vitrectomy," Retina, vol. 26, no. 7, pp. 830-831, 2006.

[63] H. Shimada, H. Nakashizuka, T. Hattori, R. Mori, Y. Mizutani, and M. Yuzawa, "Incidence of endophthalmitis after 20- and 25-Gauge vitrectomy. Causes and prevention," Ophthalmology, vol. 115, no. 12, pp. 2215-2220, 2008.

[64] A. Y. H. Hu, J. L. Bourges, S. P. Shah et al., "Endophthalmitis after pars plana vitrectomy: a 20- and 25-gauge comparison," Ophthalmology, vol. 116, no. 7, pp. 1360-1365, 2009.

[65] J. O. Mason, J. J. Yunker, R. S. Vail et al., "Incidence of endophthalmitis following 20-gauge and 25-gauge vitrectomy," Retina, vol. 28, no. 9, pp. 1352-1354, 2008.

[66] J. K. Chen, R. N. Khurana, Q. D. Nguyen, and D. V. Do, “The incidence of endophthalmitis following transconjunctival sutureless 25- vs. 20-gauge vitrectomy," Eye, vol. 23, no. 4, pp. 780-784, 2009.

[67] K. Matsuyama, K. Kunitomi, M. Taomoto, and T. Nishimura, "Early-onset endophthalmitis caused by methicillin-resistant Staphylococcus epidermidis after 25-gauge transconjunctival sutureless vitrectomy," Japanese Journal of Ophthalmology, vol. 52, no. 6, pp. 508-510, 2008.

[68] N. Acar, Y. B. Unver, T. Altan, and Z. Kapran, "Acute endophthalmitis after 25-gauge sutureless vitrectomy," International Ophthalmology, vol. 27, no. 6, pp. 361-363, 2007.

[69] H. M. Bahrani, A. A. Fazelat, M. Thomas et al., "Endophthalmitis in the era of small gauge transconjunctival sutureless vitrectomymeta analysis and review of literature," Seminars in Ophthalmology, vol. 25, no. 5-6, pp. 275-282, 2010.

[70] R. Keshavamurthy, P. Venkatesh, and S. Garg, "Ultrasound biomicroscopy findings of $25 \mathrm{G}$ Transconjuctival sutureless (TSV) and conventional (20G) pars plana sclerotomy in the same patient," BMC Ophthalmology, vol. 6, article 7, 2006.

[71] A. Singh, J. A. Chen, and J. M. Stewart, "Ocular surface fluid contamination of sutureless 25-gauge vitrectomy incisions," Retina, vol. 28, no. 4, pp. 553-557, 2008.

[72] A. Kreiger, "Sclerotomy complications following pars plana vitrectomy," British Journal of Ophthalmology, vol. 85, no. 1, pp. 121-122, 2001.

[73] G. Y. Fujii, E. De Juan, M. S. Humayun et al., "A new 25-gauge instrument system for transconjunctival sutureless vitrectomy surgery," Ophthalmology, vol. 109, no. 10, pp. 1807-1812, 2002.

[74] T. A. Meredith, "Antimicrobial pharmacokinetics in endophthalmitis treatment: studies of ceftazidime," Transactions of the American Ophthalmological Society, vol. 91, pp. 653-699, 1993.

[75] S. D. M. Chen, Q. Mohammed, B. Bowling et al., "Vitreous wick syndrome-a potential cause of endophthalmitis after intravitreal injection of triamcinolone through the pars plana," American Journal of Ophthalmology, vol. 137, no. 6, pp. 1159-1161, 2004.

[76] F. U. Huamonte, M. N. Cyrlin, H. H. Tessler, and M. F. Goldberg, "Retinopathy in diabetes associated with sarcoidosis," Annals of Ophthalmology, vol. 12, no. 11, pp. 1290-1297, 1980.

[77] G. J. Jaffe and T. C. Burton, "Progression of nonproliferative diabetic retinopathy following cataract extraction," Archives of Ophthalmology, vol. 106, no. 6, pp. 745-749, 1988.

[78] G. J. Jaffe, T. C. Burton, E. Kuhn, A. Prescott, and A. Hartz, "Progression of nonproliferative diabetic retinopathy and visual outcome after extracapsular cataract extraction and intraocular lens implantation," American Journal of Ophthalmology, vol. 114 , no. 4, pp. 448-456, 1992.
[79] A. Pollack, S. Dotan, and M. Oliver, "Progression of diabetic retinopathy after cataract extraction," British Journal of Ophthalmology, vol. 75, no. 9, pp. 547-551, 1991.

[80] H. Cheng and S. L. Franklin, "Treatment of cataract in diabetics with and without retinopathy," Eye, vol. 2, no. 6, pp. 607614, 1988.

[81] H. Schatz, D. Atienza, H. R. McDonald, and R. N. Johnson, "Severe diabetic retinopathy after cataract surgery," American Journal of Ophthalmology, vol. 117, no. 3, pp. 314-321, 1994.

[82] I. A. Cunliffe, D. W. Flanagan, N. D. L. George, R. J. Aggarwaal, and A. T. Moore, "Extracapsular cataract surgery with lens implantation in diabetics with and without proliferative retinopathy," British Journal of Ophthalmology, vol. 75, no. 1, pp. 9-12, 1991.

[83] T. Y. Y. Lai, A. K. H. Kwok, D. S. C. Lam, P. Bhende, S. Dev, and J. S. Pulido, "Progression of diabetic retinopathy after endophthalmitis," Ophthalmology, vol. 107, no. 4, pp. 619$621,2000$. 


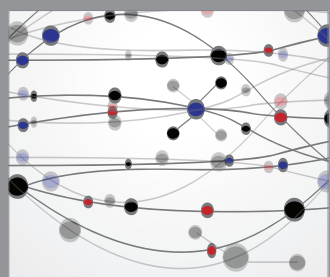

The Scientific World Journal
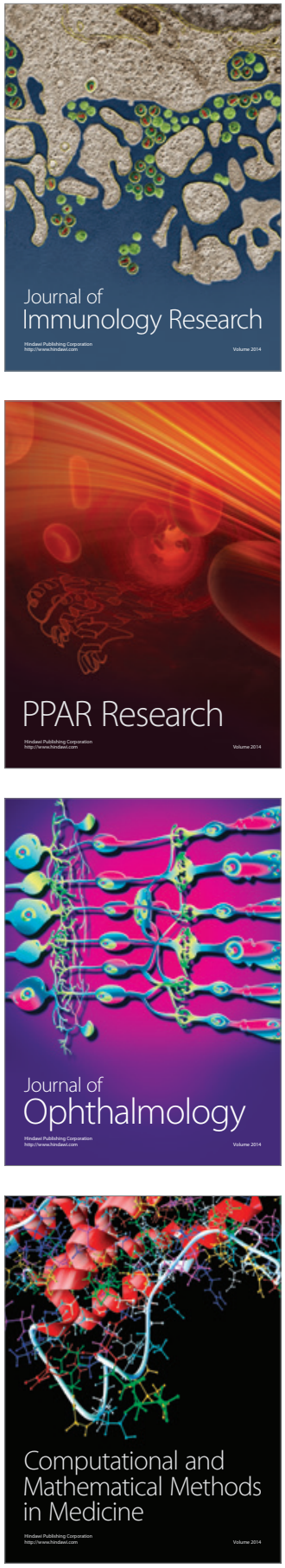

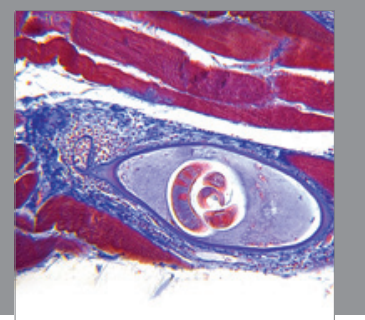

Gastroenterology

Research and Practice
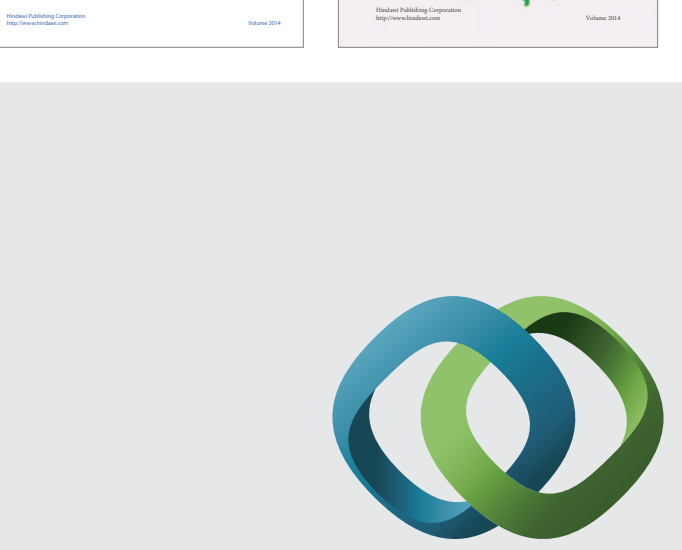

\section{Hindawi}

Submit your manuscripts at

http://www.hindawi.com
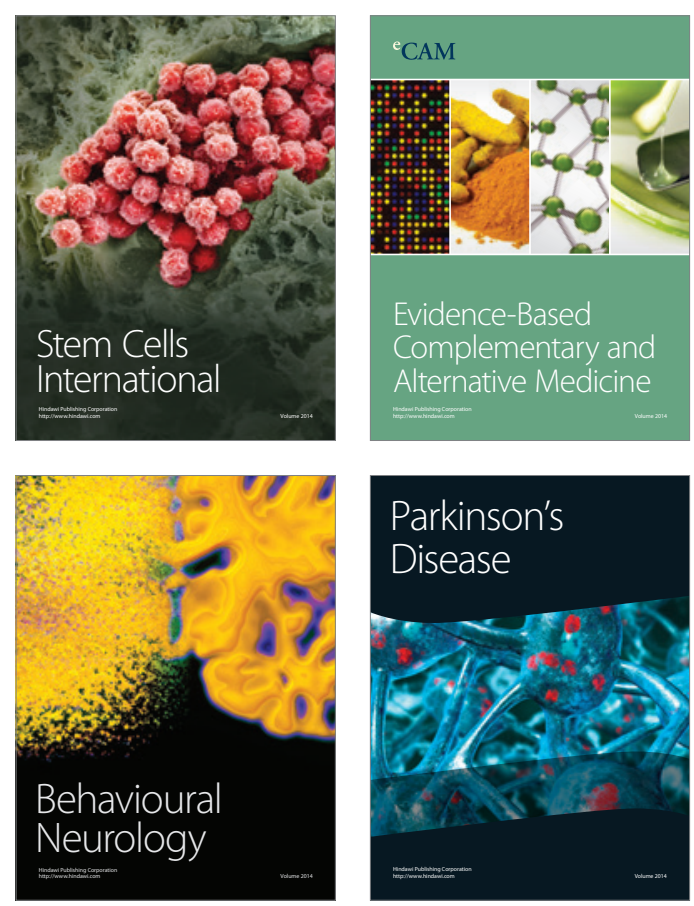

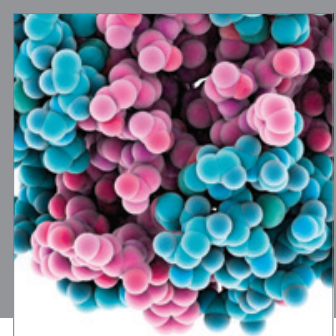

Journal of
Diabetes Research

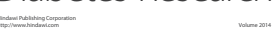

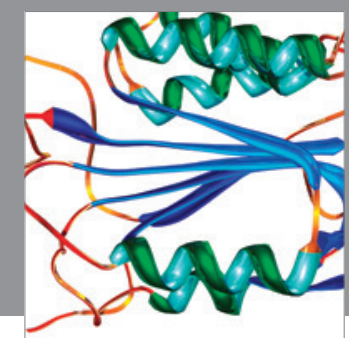

Disease Markers
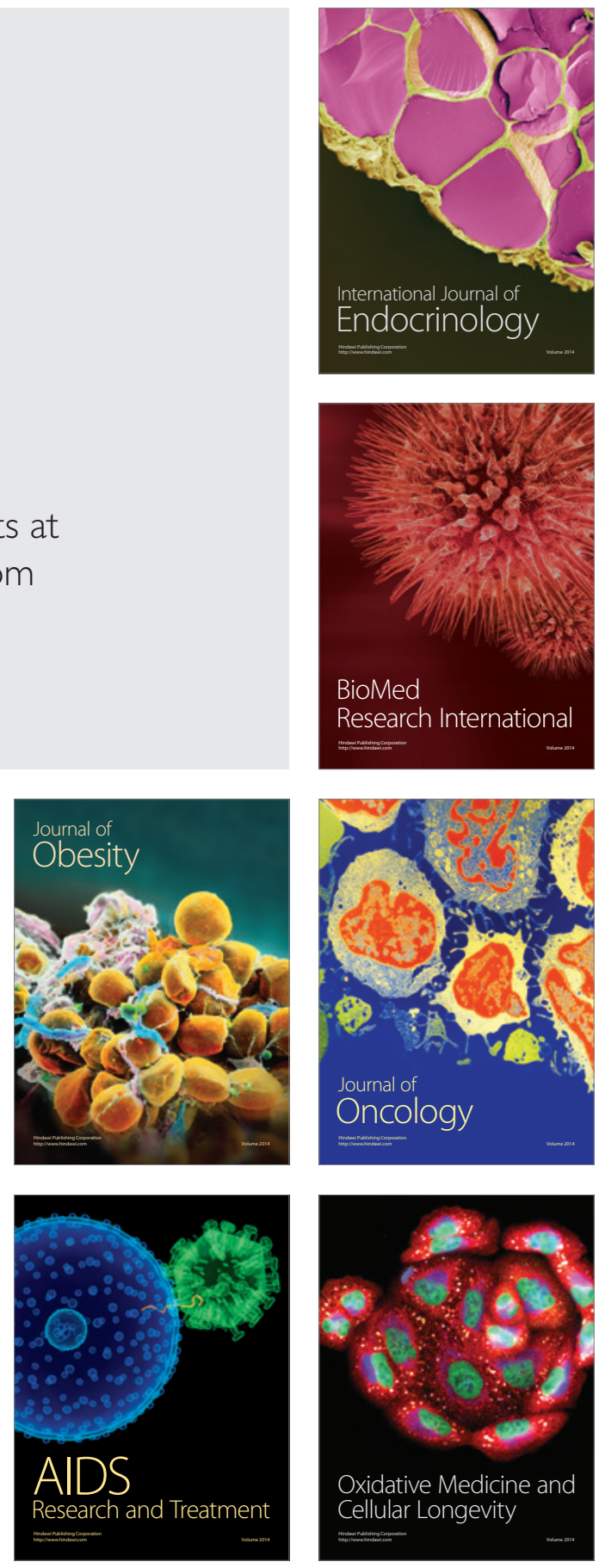